\title{
Application of static masking technique in Magnetron Sputtering Technology for the production of linearly variable filters
}

Thomas Begou ${ }^{1}$, Frédéric Lemarquis ${ }^{1}$, Antonin Moreau ${ }^{1}$, Fabien Lemarchand ${ }^{1}$, Holger Reus ${ }^{2}$, Detlef Arhilger $^{2}$, Harro Hagedorn ${ }^{2}$ and Julien Lumeau ${ }^{1, *}$

${ }^{1}$ Aix Marseille Univ, CNRS, Centrale Marseille, Institut Fresnel, F-13013 Marseille, France

${ }^{2}$ Bühler Leybold Optics, Alzenau, Germany

*julien.lumeau@fresnel.fr

\begin{abstract}
Variable filters are key components for compact spectral imagers. In this paper, we present a method for the fabrication of linearly variable filters based on Bühler HELIOS machine (plasma assisted reactive magnetron sputtering). These filters are obtained by producing a variation of the thickness of all the layers of the coating, using adapted masks placed in between the sputtering targets for the low and high refractive index materials and the substrates. Variable band pass filter from $550 \mathrm{~nm}$ up to $1000 \mathrm{~nm}$ is demonstrated.
\end{abstract}

\section{Keywords}

Optical coatings, optical interference filters, linearly variable filters, deposition technology, magnetron sputtering

\section{Introduction}

Variable filters, in which the band pass central wavelength shifts along one direction of the component, is a promising way to simplify and to miniaturize spectral imaging systems which is of high interest especially for space applications where weight and compactness are of prime need. In fact, by combining a variable filter and a 2D array detector (CCD, CMOS camera, ...), it is possible to realize simultaneously spectral analysis and imaging using pushbroom technique [1]. 
From the design point of view, the possibly to obtain variable filters relies on the dependence of the spectral properties of a multilayer coating with the thicknesses of some of its layers, if not all. In the particular case of a Fabry-Perot filter, formed with two metallic mirrors, a simple change of the thickness of the cavity layer provides a shift of its centering wavelength. This simple structure has the advantage to naturally give broad rejection bands, but is not adequate to provide sharp transition band-pass and leads to high absorption losses. To improve this last point, a solution consists to use the so-called induced transmission filter approach where a metallic layer is placed at the minimum of the electric field distribution in the cavity of dielectric Fabry-Perot filter [2-4]. However, the only way to produce a filter with arbitrary specified properties in terms of rejection, width and sharpness consists to use a standard all-dielectric approach formed with a multi-cavity Fabry-Perot structure associated with additional dielectric short and long wavelength pass blocking filters. In that case, the thickness of all the layers must be tuned by a common factor, resulting in a proportional wavelength shift, to produce a variable filter $[5,6]$.

Obviously, increasing the performance requires to increase the design complexity, and namely the layer count: a few layers for a metallic Fabry-Perot structure, to a few tens for an induced transmission filter, and to more than one hundred for an all dielectric solution.

There are mainly two technologies that have been investigated to produce variables filters. One consists in combining thin-film deposition with lithography and etching, but is limited to metal-dielectric approach or single cavity all-dielectric Fabry-Perot filters to avoid multiple etching processes [7]. The second consists in producing masks that are inserted into the deposition machine in order to generate, by the mean of a relative motion between the masks and the substrates, large and controllable thickness gradients.

Several masking geometries exist, resulting in different characteristics in terms of deposition rate and mass-production capability, but that also concern the curvature of iso-thickness lines on variable filters. Up to now, we only mentioned the thickness gradient as the main characteristic of a variable filter. However, one can define a "uniform" direction that is perpendicular to the "non-uniform" gradient direction, and iso-thickness lines along this uniform direction should ideally be perfectly straight. To achieve such a result, the substrate and the mask must have a relative translation movement, necessarily back and forth, the substrate being completely hidden behind the mask at both extremities. The main consequence of such a translation geometry is that only one filter is likely to be manufactured at a time, moreover with a very low deposition rate $[2,3]$.

On the contrary the use of a continuous rotation movement, which is compliant with most deposition machines equipped with a rotary substrate holder, allows a maximum deposition rate and the manufacturing of several filters at a time, but necessarily results in circular iso-thickness lines, the radius of curvature being equal to the distance to the rotation axis. 
These two cases are detailed in reference [8] which also reports on a more complex solution mixing both rotation and translation movements in order to benefit from the advantages of each of these exclusive solutions. The impact of the curvature of iso-thickness lines completely depends on filters applications. In case the filter is directly associated with a detector, a wavelength calibration can be performed to take account of this curvature. But it might be more critical if filters are associated with other dispersive elements [9].

In our case, we are interested in the manufacturing of all dielectric variable filters that typically cover the spectral range of silicon detectors $(400-1100 \mathrm{~nm})$. Although this paper does not deal with the manufacturing of such filters, preliminary design studies allowed us to estimate the required layer count to about 140, for a total thickness up to $18 \mu \mathrm{m}$. Among the deposition machines that are available at Institut Fresnel, the Bühler HELIOS machine is considered as the most appropriate. First, its deposition process is highly stable, and particularly interesting for the deposition of complex coatings with a high layer count, as proved by the filters we manufactured for the IDEFIX, TARANIS or 3MI projects [10-12] with more than 100 layers and $25 \mu \mathrm{m}$ thick. Second, the HELIOS rotating substrate holder has a large diameter, between 460 and $740 \mathrm{~mm}$, which gives, according to the position of the gradient on the substrate relatively to the substrate holder and using a simple masking technique, a $700 \mathrm{~mm}$ radius of curvature for variable filters, this value being large enough for our requirements.

The goal of this paper is to describe the masking system we developed for the manufacturing of variable filters with this machine, and the tests we performed to demonstrate the validity of the proposed approach. We will validate this process with the deposition of single layers of high and low refractive index materials $\left(\mathrm{Nb}_{2} \mathrm{O}_{5}\right.$ and $\left.\mathrm{SiO}_{2}\right)$ and of two cavities band pass filters $[13,14]$.

\section{Experimental set up to create a gradient}

\subsection{Deposition system description}

The deposition technology used in this work is plasma assisted reactive magnetron sputtering (PARMS) with a HELIOS machine from Bühler Leybold Optics [15]. The deposition parameters (for sputtering and plasma assistance) and geometry (targets, uniformity mask...) are identical to that are used with the standard configuration of a HELIOS machine. The only difference is described hereafter and consists in adding masks inserts within the actual shutter. The filters presented in the following parts are made using $\mathrm{Nb}_{2} \mathrm{O}_{5}$ and $\mathrm{SiO}_{2}$ respectively for high and low index materials.

\subsection{Masking technology}

In the Helios machine, substrates are located on a turntable, a few centimeters below the sputtering targets. The deposition is consequently intermittent and occurs only when a substrate is under a 
sputtering target, which approximately corresponds to a $120 \times 120 \mathrm{~mm}^{2}$ area for each target. The deposited thickness during each turn is then proportional to the time required for the substrate to rotate in front of the target. If a mask is positioned between the target and the substrate, the deposited thickness is then proportional to the time required for the substrate to rotate in front of the open area of the mask, or in other words to the length of this open area. In its standards configuration, such masks are used to insure the best thickness radial uniformity (generally much better than $1 \%$ over $100 \mathrm{~mm}$ diameter). The idea developed for this study consists in developing masks that will produce an opposite effect, and generate a significant radial thickness gradient.

The developed technique consists in keeping uniformity masks below the target which guarantee a starting uniform distribution of sputtered material and to add new masks as close as possible to the substrates to control the deposition time of material along the radial direction on the substrate and thus generate the thickness gradient (Figure 1). However, for convenience reasons, we decided to implant these new masks directly on the shutter that is placed in between the targets and the substrates. But due to the few millimeters between the masks and the substrates, the design of the masks requires careful design and mounting.

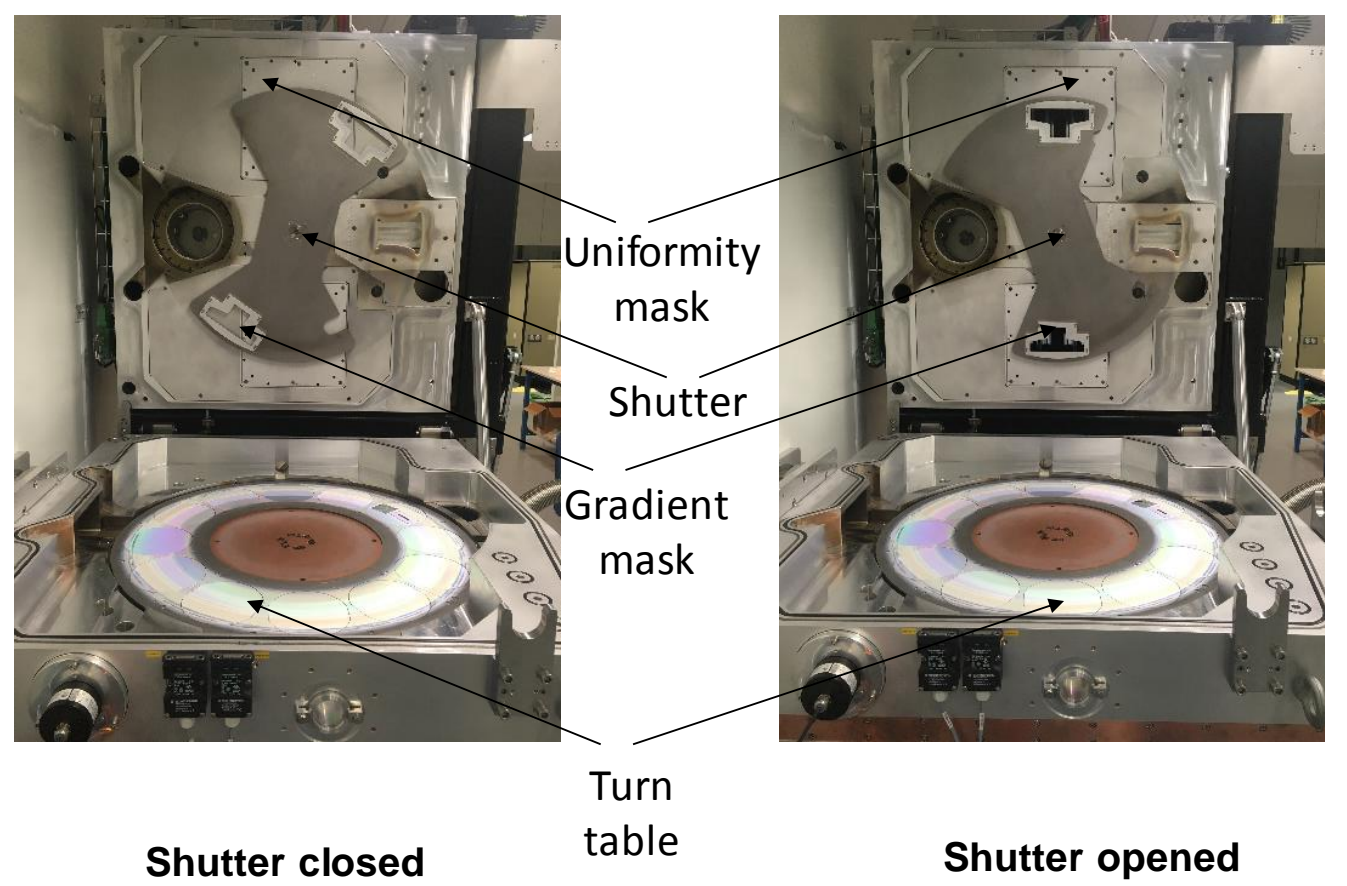

Fig. 1. Masking set up installed on the HELIOS deposition machine. At the bottom one can see the turntable where samples are inserted. On top, one can see the masks installed on the shutter position right in front of the magnetron sources. When lid is closed and turntable is rotating, each samples moves in front of each magnetron source and a thickness gradient is produced. 
A new type of shutter and masks was designed, fabricated and then installed on the HELIOS machine $[13,14]$. The shutter is made of titanium, like the original shutter, to limit the deformation related to thermal effects while the masks are made of aluminum to allow an easier machining during the development phase. Then, they should also be made of titanium as well as the fixing screws to further minimize thermal effects. The control programs of the machine have also been adapted to allow the use of the mask with new adapted positions.

\subsection{Principle for the generation of a variable filter}

In order to validate the principle of a thickness gradient generation, we designed a first set of masks, as illustrated in the Figure 2. The masks have 3 distinct zones:

- A uniform $46 \mathrm{~mm}$-long and $55 \mathrm{~mm}$-wide area to perform in-situ optical monitoring. This area allows to control the thickness deposited in the region having the thinnest material thickness (zone number 1 in figure 1).

- An area which width varies by a factor of 3 from 55 to $165 \mathrm{~mm}$ over a distance of $10 \mathrm{~mm}$. It is this area that will be used to generate a thickness gradient with a ratio close to or equal to 3 (zone number 2 in figure 1 ).

- Another uniform area $19 \mathrm{~mm}$-long, $165 \mathrm{~mm}$-wide to avoid the shading effects associated with the edge of the mask (zone number 3 in figure 1).
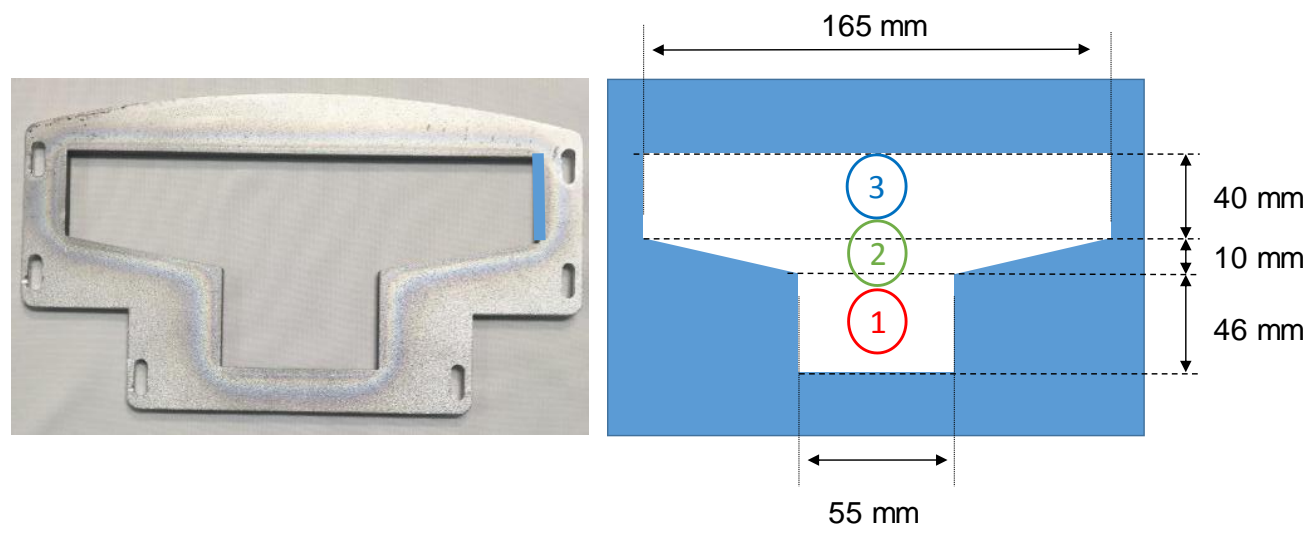

Fig. 2. Photography and schematics of the first mask designed to generate variable filter.

The maximum width of the masks is fixed by the wider aperture of the material sources $(165 \mathrm{~mm})$ in order to keep the deposition rate at its maximum, close to the one in classical uniform configuration of the HELIOS machine $\left(\approx 0.4-0.5 \mathrm{~nm} \cdot \mathrm{s}^{-1}\right.$ for both materials). 


\section{Deposition of single layers with variable thicknesses}

In order to precisely characterize the generated gradient, we have deposited, through the gradient mask described previously in figure 2 :

- a 5 quarter-wave, at $500 \mathrm{~nm}$, (i.e. $\sim 272 \mathrm{~nm}$ thick) single layer of $\mathrm{Nb}_{2} \mathrm{O}_{5}$ on a silica substrate,

- a 5 quarter-wave, at $500 \mathrm{~nm}$, (i.e. $\sim 428 \mathrm{~nm}$ thick) single layer of $\mathrm{SiO}_{2}$ on a silica substrate previously coated with a uniform 5 quarter wavelength at $500 \mathrm{~nm}$ layer of $\mathrm{Nb}_{2} \mathrm{O}_{5}$.

The local transmission has been measured using a custom set-up that allows to map the transmission over the sample aperture [16]. The measurement has been performed along the $\mathrm{x}$ direction over 93 $\mathrm{mm}$, with a step of $500 \mu \mathrm{m}$ and a beam size of approximately $100 \mu \mathrm{m}$. To extract the thickness profile of both materials, reverse engineering has been used for each measured spectral transmission curve. The estimated error on this calculation is in the order of one nanometer. Figure 3 illustrates the absolute and relative evolution of the physical thicknesses of both materials along the $x$ direction. At both extremities of the mask, sharp variations of the thickness can be noticed as shadowing effects associated with the mask. Then two distinct regions can be observed: one between 25 and $60 \mathrm{~mm}$ associated with the uniform zone of the mask and one between 65 and $80 \mathrm{~mm}$ associated with the gradient zone of the mask. Let us now analyze these two regions into details.
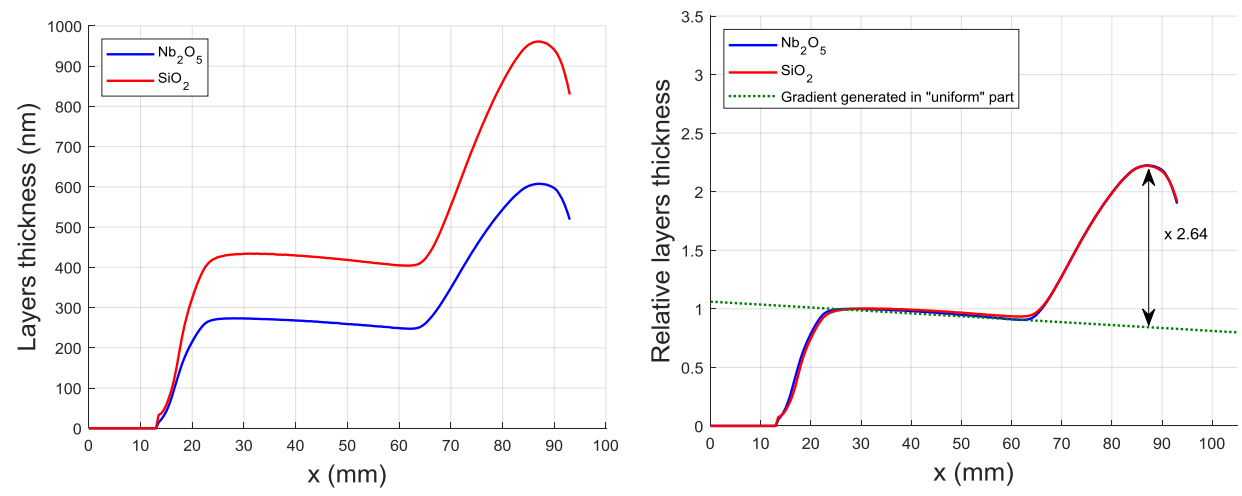

Fig. 3. Physical (left) and relative (right) thickness profiles for single layers of $\mathrm{SiO}_{2}$ and $\mathrm{Nb}_{2} \mathrm{O}_{5}$.

Firstly, it can be noted the same mask gives almost identical thickness gradient for both materials, this last condition being required to guarantee the production of high performance variable filters. As a consequence, two identical masks can be used for both materials. Secondly, a comparison between the measured relative thicknesses evolutions and the expected variation derived from the shape of the masks is plotted in figure 3, and one can see that a mismatch exists. Indeed, the gradient of thickness is measured over a $18.5 \mathrm{~mm}$ range instead of the expected $10 \mathrm{~mm}$ and the ratio between the thickest and the thinnest regions is 2.22 instead of 3 . This mismatch is probably due to shadowing effect linked to the distance between the mask and the substrate. This reveals a limitation of the considered masks 
geometry to achieve large gradients and large deposition rates. We observed that by decreasing the overall aperture of the mask, it is possible to better approach the expected gradient but with a significant decrease of the deposition rate. We decided in this work to keep this mask geometry and thus we opted for the calculation of calibration parameters to account for this mismatch.

A second mismatch can be seen also in the uniform region devoted to optical monitoring. The thickness was indeed expected to be constant, while one can observe a slight decrease (-0.25\%.mm-1). To achieve such a result, the mask should have in this region the shape of an angular sector centered on rotation axis of the substrate holder. For an easier manufacturing of this test mask, this shape has been replaced by parallel edges, resulting in a slightly decreasing thickness, which is not considered as critical at this point.

Finally, considering this tooling factor but also due to the gradient observed in the supposedly uniform region in the optical monitoring region, one can show that the real gradient produced by the increase of the aperture of the mask is not 2.22 but closer to 2.64 (right part in Figure 3). Moreover, as seen in Figure 4 , the deviation towards a linear gradient is less than $\pm 2 \%$ over a $14 \mathrm{~mm}$ range.

Despite these discrepancies, one can see that a ratio of 2 on the thickness can be achieved over a $12 \mathrm{~mm}$ aperture, which is within what is commonly proposed by linearly variable filters manufacturers $[17,18]$.

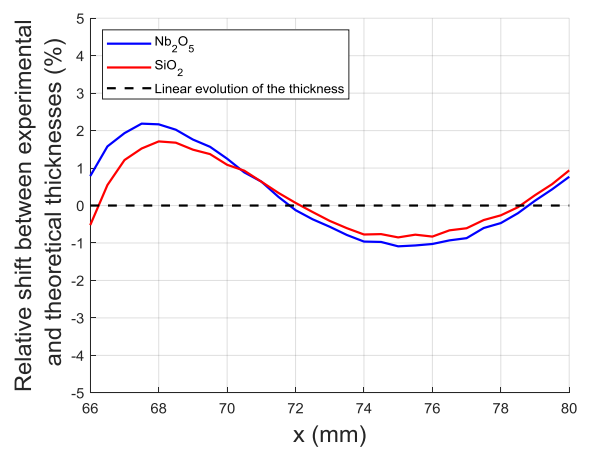

Fig. 4. Relative shift between the experimental and theoretical thickness gradient for single layers of $\mathrm{SiO}_{2}$ and $\mathrm{Nb}_{2} \mathrm{O}_{5}$ in the linear gradient region (right).

\section{Theoretical approach}

One specificity of the proposed approach is that, due to the machine geometry, two distinct masks are required to produce the gradient of thickness, one for each material source. Therefore, to produce high quality optical filters, it is mandatory to be able to precisely position the masks in a way that both the 
high and low index materials thickness gradients will be perfectly superimposed on the substrates to be coated. To allow a precise relative positioning of the masks a preliminary manufacturing test has been planned with a specific 2-cavity band pass filter, one cavity being made of high index material, the other one of low index material: H L 2H L H L H $2 \mathrm{~L} \mathrm{H}$. In case the two masks are miss-positioned, resulting in different thickness gradients for both materials, the centering wavelengths associated to each cavity should gradually sweep along the variable direction, resulting in a final distortion and collapse of the band pass profile. Figure 5 gives the spectral transmission profile of such a filter, centered at wavelength $500 \mathrm{~nm}$ and modeled using standard matrix formalism [19].

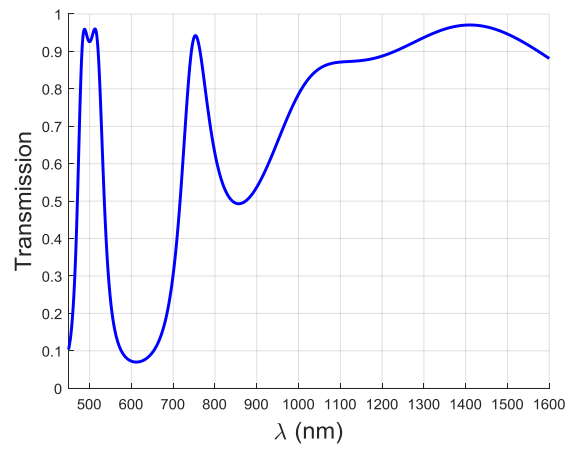

Fig. 5. Transmittance of the double cavity Fabry-Perot when centered at $500 \mathrm{~nm}$.

Assuming that the film thickness is directly proportional to the mask width, we can calculate the evolution of the spectral profile of the filter. This evolution is given in figure 6 .
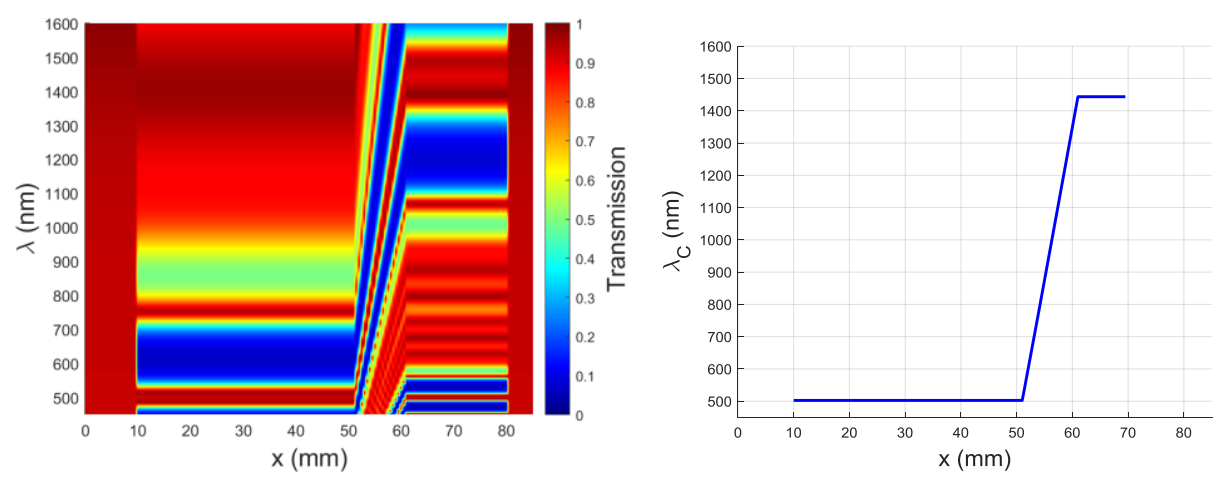

Fig. 6. Spectral evolution of the transmission (left) and of the central wavelength $\lambda C$ (right) of the double cavity Fabry-Perot filter along the $x$ direction.

The representation chosen in the left part of figure 6 allows to plot on the same graph the evolution of the transmission in a color level scale as a function of the wavelength and the $x$ position. Below the 46 $\mathrm{mm} x$-position, the spectral transmission profile of the filter is constant and identical to the one plotted in figure 5. From $x=46 \mathrm{~mm}$ to $56 \mathrm{~mm}$, we can see (right part of figure 6) that the central wavelength of 
the filter varies from $500 \mathrm{~nm}$ to $1443.5 \mathrm{~nm}$. Despite an increase of the thickness by a factor of 3 from the narrowest to widest part of the mask, the filter central wavelength only increases by a factor of 2.89 . This is due to the fact that the central wavelength of a filter is not linked to physical thickness but to optical thickness and the decrease of refractive index with wavelength (dispersion).

These calculations were made considering the same thickness gradients for the high and low refractive index materials. Let us now consider the result considering some miss-positioning between the two masks. Considering the projection of the masks image for both targets on the sample to be coated, a misalignment $(\Delta x)$ between the masks on both targets, for high and low refractive index materials shifts the two gradients of material along the $x$ direction on the sample. A parametrical simulation has then been performed by varying $\Delta x$ from $-2.5 \mathrm{~mm}$ to $2.5 \mathrm{~mm}$. These values were chosen as they seem to be representative of the mechanical tolerances associated with the masks and the opened shutter on the machine (see figure 1 and 2). It can be easily shown that except at the gradient boundary, linear gradient is kept when masks are misaligned. But the most visible effect of a mask misalignment concerns the spectral performance of the filter, especially the shape of the band pass. Figure 7 gives the spectral dependence of the transmission when the filter is centered at $650 \mathrm{~nm}$ for different $\Delta \mathrm{x}$ misalignment values. As can be seen, the transmittance level in the band pass is severely affected and decreases as a function of the shift between both masks, from $100 \%$ down to $50 \%$, whether this shift is positive or negative. An asymmetry of the band pass is also observed and this asymmetry depends on the sign of $\Delta x$. If the shift is negative, the amplitude of the first peak is higher than the one of the second peak. An opposite effect appears when the shift is positive, i.e. the amplitude of the second peak is higher than the one of the first peak. This effect is used to determine the relative position of the two masks: the position of the maximum (first or second peak) providing an information on what gradient starts first and the relative amplitude of the maxima of transmission, the exact distance between these two masks.
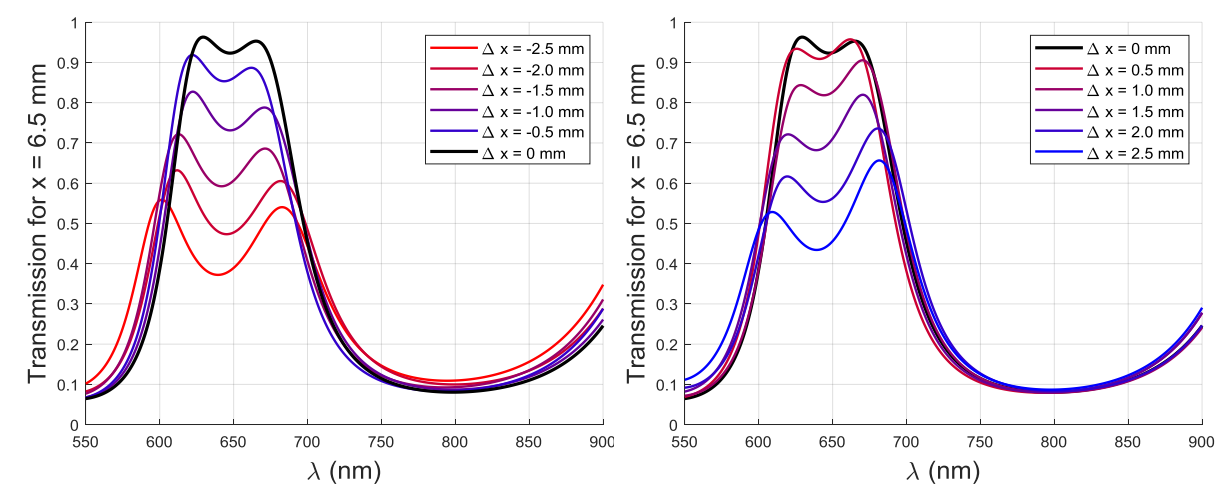

Fig. 7. Spectral evolution of the transmission of the double cavity Fabry-Perot filter when it is centered at $650 \mathrm{~nm}$ with different negative (left) and positive (right) values of $\Delta \mathrm{x}$. 


\section{Deposition of a band pass Fabry-Perot filter}

\subsection{Mask alignment procedure}

Gradient masks were first set in a position on the shutter that should be close to the optimal one. Then, the double cavity Fabry-Perot filter was deposited. To check the relative positions of both masks, the local transmission was measured on one point in the gradient region. As seen on the left part of figure 11 , this measurement was compared with the theoretical transmission calculated with no shift $\Delta x$ between both masks. The maximum of the measured transmission is less than that of the theoretical one and dissymmetry between both maxima is observed on experimental data. As demonstrated previously, this effect can be explained by a shift between the relative positions of both masks. If a -2 $\mathrm{mm}$ shift $\Delta \mathrm{x}$ is added in the simulation, a much better agreement between experimental and theoretical transmission curves is achieved, as seen on the right part of the figure 12 . The residual mismatch between the curves can be easily explained by measurement uncertainties and especially the averaging of the variable transmittance over the measurement spot, which is not taken into account for the calculated profile.
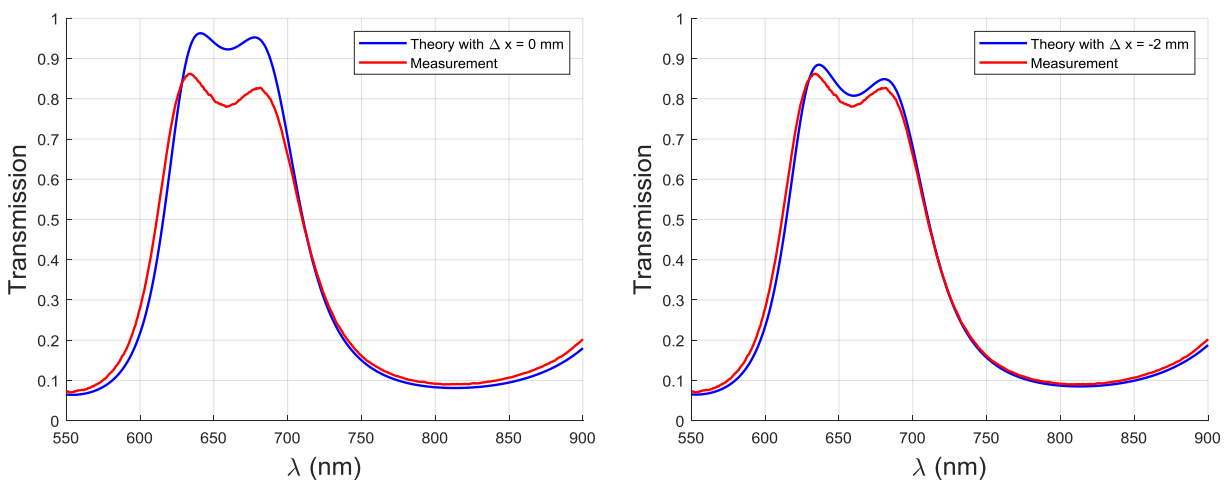

Fig. 12. Comparison between the local transmission measured on the sample and the theoretical one for different values of the shift between both masks used in the simulation $\Delta x$. (on the left part $\Delta x=0 \mathrm{~mm}$ and on the right part $\Delta \mathrm{x}=-2 \mathrm{~mm}$ ).

To align the masks correctly, a $+2 \mathrm{~mm}$ shift was then physically applied on the position of the gradient mask under the low index material target. The same deposition of double cavity Fabry-Perot was carried out and the local measurement of transmission was again performed within the same region. The transmission is illustrated in figure 13 and, for this configuration, the experimental transmission matches with theory, confirming that the misalignment between the two masks has been corrected. These results therefore show that the proposed method allows to accurately predict the relative misalignment between the two masks and careful analysis allows to conclude that a precision of $\pm 100 \mu \mathrm{m}$ between the 
position of the two masks can be achieved. Simulation of various band pass filters showed that such a mismatch is small enough to secure high performance linear band pass filters.

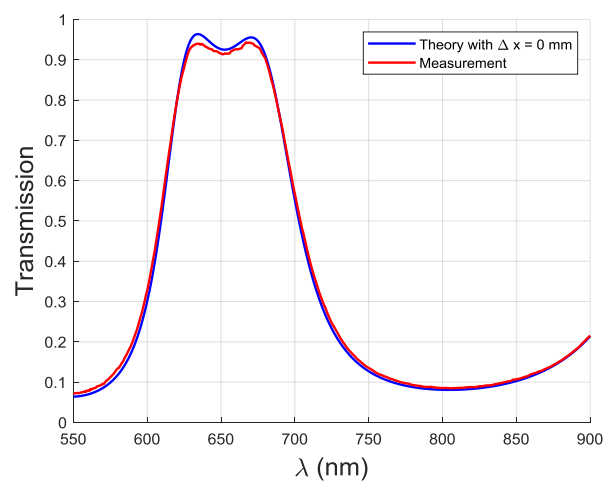

Fig. 13. Comparison between the local transmission measured on the sample and the theoretical one with a $+2 \mathrm{~mm}$ shift of the mask under the low index material target.

\subsection{Linearly variable filter characterization}

Up to now, we only looked at one transmission spectrum measured in one arbitrarily chosen position in the gradient zone, for alignment purpose. Since the last profile shows that the alignment is now correct, this last filter can be considered as representative of our capability to manufacture variable filters. We can now focus on the characterization of its performance within the whole gradient zone. To extract the performance of the filter, the local transmission spectra was measured within the uniform and gradient zones with the same set up described in section 3 and the upper left par of figure 14 gives the spectral evolution of the transmission as a function of $x$. The left part of the figure 14 is a 3D plot of the evolution of the measured transmission as a function of the wavelength and the $x$ position. Globally, the shape of this evolution is relatively close to the one plotted in figure 3. Similarly, to the spatial evolution of the transmission spectra measured in single layers, we observe some shadowing effects at the gradient masks openings boundaries (i.e. for $x$ position close to zero and 60) resulting in sharp fluctuations of the transmission as well as two regions with either a uniform or linear gradient of the central wavelength of the filter. 

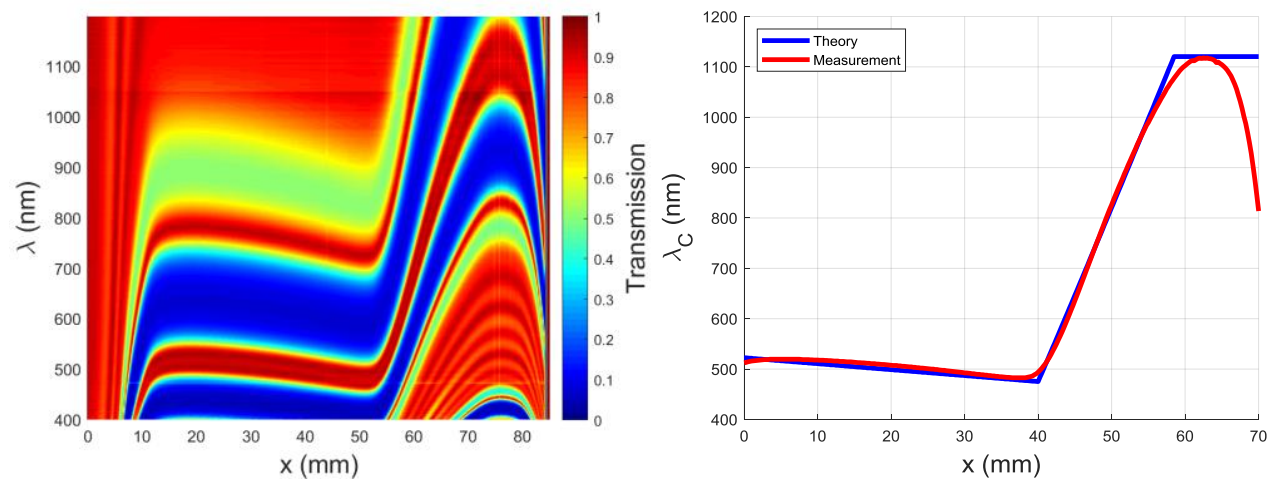

Fig. 14. Measured spectral evolution of the transmission (left) and of the measured and theoretical central wavelength $\lambda C$ (right) of the double cavity Fabry-Perot filter along the $x$ direction (linearly variable filter zone is in the blue part).

To accurately characterize the gradient zone, the central wavelength of the band pass was extracted for all the $\mathrm{x}$ positions and is plotted in the right part of figure 13. To compare this evolution with the theoretical one, the evolution of the central wavelength of the filter was simulated with the corrected parameters defined with single layers (see Figure 3). Using this model, one can see a very good agreement between theory and experiment confirming that the parameters used to model single layers allows an accurate modeling of more complex structures. Finally, as seen in the top left of figure 15, if we remove the transition zones within the gradient region, a filter with a band pass linearly varying from $550 \mathrm{~nm}$ to $1000 \mathrm{~nm}$ over a $12 \mathrm{~mm}$ distance (i.e. $37.5 \mathrm{~nm} . \mathrm{mm}-1$ ) was realized. The measured and theoretical spectral transmissions are also plotted for different $x$ position on the filter, in figure 15, with a very good agreement from $500 \mathrm{~nm}$ to $1050 \mathrm{~nm}$ that is the wavelength range of the variable filter. 

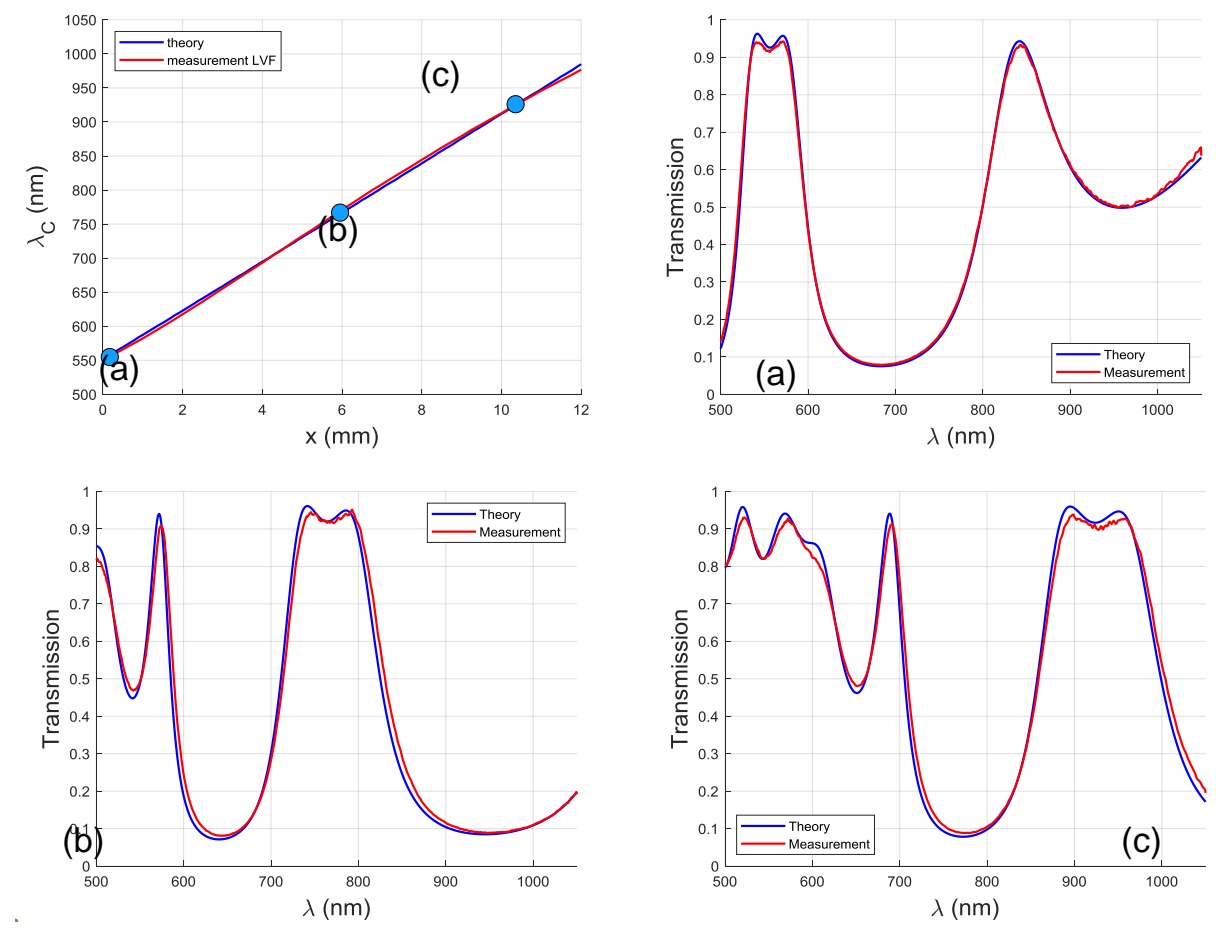

Fig. 15. Measured and theoretical central wavelength $\lambda C$ (top left) of the double cavity Fabry-Perot filter along the $\mathrm{x}$ direction and measured and theoretical spectral transmission for different $\mathrm{x}$ position $(\mathrm{a}, \mathrm{b}$ and c).

\section{Conclusions}

We have demonstrated in this paper an efficient method for variable thickness generation, compatible with a Bühler HELIOS type deposition machine, and allowing the fabrication of complex filters. Due to the machine geometry, this method relies on the use of two gradient masks, one for each material, that are fixed on the shutter. Because of shadowing effects resulting from the distance that exists between the shutter and the substrates to coat, the thickness gradient cannot be obviously related to the mask shape. For this purpose, measurement performed on variable single layers helped us to define a correction factor that allows now to design masks for a specified thickness gradient. We also developed a method that guarantees an accurate relative positioning of the two masks, based on the deposition of a simple 9-layer two-cavity Fabry-Perot filter, each material being used to form one of these cavities. The wavelength shift measured on this filter is perfectly consistent with the results observed on single layers, the centering wavelength being approximately doubled within $13 \mathrm{~mm}$. 
All the required tools seem now ready for the manufacturing of a more efficient all-dielectric variable filter, with a much more complex design since more than one hundred layers are required for a narrow band filter covering a similar spectral width with a reasonable rejection level.

\section{Declarations}

\section{Funding}

The authors would like to acknowledge the French space agency (CNES) for the funding of this work.

\section{Conflicts of interest/Competing interests}

Not applicable

Availability of data and material

Data can be provided upon request

Code availability (software application or custom code)

Not applicable

\section{Authors' contributions}

All the authors contributed equally to the work

Additional declarations for articles in life science journals that report the results of studies involving humans and/or animals

N/A

Ethics approval (include appropriate approvals or waivers)

N/A

Consent to participate (include appropriate statements)

N/A

Consent for publication (include appropriate statements)

N/A 


\section{References}

1. P. Mouroulis, R. O. Green, and T. G. Chrien, "Design of pushbroom imaging spectrometers for optimum recovery of spectroscopic and spatial information", Appl. Opt. 39 (13), 2210-2220 (2000).

2. A. Piegari and J. Bulir, "Variable narrowband transmission filters with a wide rejection band for spectrometry," Appl. Opt. 45, 3768-3773 (2006).

3. A. Piegari, J. Bulir, and A. Krasilnikova Sytchkova, "Variable narrow-band transmission filters for spectrometry from space. 2. Fabrication process, " Appl. Opt. 47, C151-C156 (2008).

4. C. Williams, G. Rughoobur, A. J. Flewitt, and T. D. Wilkinson, "Single-step fabrication of thin-film linear variable bandpass filters based on metal-insulator-metal geometry," Appl. Opt. 55, 9237-9241 (2016).

5. F. Lemarquis, L. Abel-Tiberini, C. Koc, "400-1000 nm all-dielectric linear variable filters for ultra compact spectrometers, " Proc. SPIE 10565, International Conference on Space Optics - ICSO 2010, 105655U (5 September 2019).

6. $\underline{\text { C.-H. Ko }}$ J.-R. Tsai, B.-J. Wang, S.-F. Lin, C.-T. Hong, and W.-H. Chiu "Visible and near-IR range linear variable filter with two-dimensional modeling ", Proc. SPIE 10746, Novel Optical Systems Design and Optimization XXI, 1074607 (17 September 2018).

7. Emadi, H. Wu, S. Grabarnik, G. De Graaf, K. Hedsten, P.Enoksson, J. Correia, and R. Wolffenbuttel,"Fabrication and characterization of IC-compatible linear variable optical filters with application in a micro-spectrometer," Sens. Actuators A162, 400-405 (2010).

8. L. Abel-Tibérini, F. Lemarquis, and M. Lequime, "Masking mechanisms applied to thin-film coatings for the manufacturing of linear variable filters for two-dimensional array detectors," Appl. Opt. 47, 5706-5714 (2008).

9. P. Bernardi, M. Bonafous, M. Motisi, J-M. Reess, J. Tanrin, D. Laubier, "Wedge filter imaging spectrometer," Proc. SPIE 10563, International Conference on Space Optics - ICSO 2014, 1056355 (17 November 2017)

10. F. Lemarquis, T. Begou, A. Moreau, J. Lumeau, "Broadband antireflection coatings for visible and infrared ranges," CEAS Space Journal 11 (4), pp.567-578 (2019).

11. T. Begou, F. Lemarchand, F. Lemarquis, A. Moreau, J. Lumeau, "High performance thin-film optical filters with stress compensation, "Journal of the Optical Society of America. A Optics, Image Science, and Vision 36 (11), pp.C113-C121 (2019).

12. T. Begou, H. Krol, D. Stojcevski, F. Lemarchand, M. Lequime, C. Grezes-Besset and J. Lumeau, "Complex optical interference filters with stress compensation for space applications", CEAS Space J 9(4), 441-449 (2017).

13. T. Begou, F. Lemarquis, A. Moreau, F. Lemarchand, H. Reus, D. Arhilger, H. Hagedorn and J. Lumeau, "Linearly variable filters fabricated by magnetron sputtering technology, " Proc. SPIE 11180, International Conference on Space Optics - ICSO 2018, 1118084 (12 July 2019); 
14. T. Begou, F. Lemarquis, A. Moreau, F. Lemarchand, H. Reus, D. Arhilger, H. Hagedorn and J. Lumeau, "Non-linearly variable filters for spectro-imaging systems, " Proceedings SPIE 11852, International Conference on Space Optics - ICSO 2020; 118521B (2021);

15. M. Scherer, J. Pistner, and W. Lehnert, "UV- and VIS Filter Coatings by Plasma Assisted Reactive Magnetron Sputtering (PARMS)," in Optical Interference Coatings, OSA Technical Digest (Optical Society of America, 2010), paper MA7.

16. L. Abel-Tiberini, F. Lemarquis and M. Lequime, "Dedicated spectrophotometer for localized transmittance and reflectance measurements, " Appl. Opt. 45(7), pp. 1386-1391 (2006).

17. https://materion.com/-/media/files/precision-optics/linearvariablefilters.pdf

18. https://www.edmundoptics.com/f/linear-variable-bandpass-filters-5fd8f505/14865/

19. H.A. Macleod, Thin-Film Optical Filters, Third Edition (Institute of Physics Publishing, 2001) 